\title{
MIGROS: A Model-Driven Transformation Approach of the User Experience of Legacy Applications
}

\author{
Luca Mainetti, Roberto Paiano, and Andrea Pandurino \\ GSA Lab, University of Salento, Via Monteroni, 73100 Lecce, Italy \\ \{luca.mainetti, roberto.paiano, andrea.pandurino\}@unisalento.it
}

\begin{abstract}
Model-driven engineering is a promising approach for the modernization of legacy applications, but there are still many issues to address, especially to obtain automatic refactoring of the User Experience (UX) of existing applications applying modern interaction paradigms as Rich Internet Application (RIA). The MIGROS tool tries to solve the hurdles for the model-driven modernization of the UX of legacy application (as implemented in Cobol CICS, Visual Basic, Power Builder, etc.). It is designed upon a set of well-known methods in the web engineering field. It is implemented as a set of Eclipse plug-ins that support reverse engineering and model transformation exploiting the OMG Architecture-Driven Modernization (ADM) technology.
\end{abstract}

\section{Introduction}

According to a 2003 report by the Aberdeen Group, investment in Legacy Applications (LAs), which account for upwards of $70 \%$ of enterprise business operations, consumes as much as $80 \%$ of software budgets. In a 2006 report, Gartner suggests that organizations need a wholesale re-architecting of the application platform, and conclude that "CIOs and their IT executives must complete enterprise platform migration by 2009." Even if the current economic crisis reduced IT budgets and slowed down the process, in the next future many organizations will start LA's modernization projects. Currently, many tools are available to convert legacy languages to new ones (e.g. from Cobol to Java). They mainly focus on the business logic and the server side of LAs. We observe a lack of approaches specifically intended to migrate the client side. To overcome this limitation, here we present a demo of the MIGROS tool chain that developers can use to automatically modernize the client side of LAs. Thanks to its MDE approach, MIGROS allows developers to refactor the User Experience (UX) of LAs during the modernization journey, taking the opportunities offered by novel interaction paradigms (i.e. RIA). MIGROS is deployed as a set of Eclipse plug-ins that support reverse engineering and model transformation adopting well-known methods in the web engineering field, and exploiting the OMG ADM technology.

Section 2 reports on key related works. Section 3 gives an overview of the MIGROS tool chain architecture and shows an example of its user interface. Finally, section 4 draws conclusions and sketches future steps. 


\section{Related Work on RIA Modeling and Generation Approaches}

Other authors have proposed the use of Model Driven Architectures to evolve web systems [4], and RIAs to unleash superior user interaction [2]. In general, existing methods lack in connecting re-design artifacts with UX requirements. If complex LAs are evolved to web systems, often the presentation layer is migrated to a RIA whereas the business logic and data are offered through a SOA [1]. At the same time the OMG's ADM Task Force (omg.adm.org) is proposing a set of modernization standards to facilitate the exchange of existing systems meta-data for various modernization tools. The OMG's Knowledge Discovery Meta-model (ISO/IEC 19506) provides a comprehensive view of application structure and data, but does not represent directly the UX, even if it is open to exchange meta-data form other models. Many RIA design methods are actually available (readers can look to [3] for a complete survey). The Object Oriented Hypermedia Design Method (OOHDM) proposes a model process for requirements modeling, conceptual modeling, navigation design, interface design, and implementation. WebML for RIAs extends the WebML method considering a well-defined separation between the client side and the server side, and a better definition of the application interface. The Rich Internet Application User eXperience (RUX) method defines the interface of an application through four levels: concepts and tasks, abstract interface, concrete interface, and final interface. UML-based Web Engineering (UWE) exploits an UML profile and integrates RUX method to provide a specification of a rich web system. OOH4RIA is a model-driven approach to design a complete RIA for the GWT framework. OOWS is an approach to develop web applications in an $\mathrm{OO}$ modeling oriented software development environment. ADRIA is an UML-based method for designing RIAs departing from the results of an objectoriented analysis. Internet Application Modeling Language (IAML) aims to provide modeling support for all of the fundamental concepts of RIAs using ECA rules, ER diagrams, and UML diagrams. Very often the same research groups that proposed design methods, also released tools to model, fast prototype, and generate web systems and RIAs. Some of these are very strictly related to our work as they use similar approaches and architectures: WebTE, WebRatio, MVC-Webflow, UWE4JSF, MagicUWE, RUX-Tool, and OIDE. Whereas these approaches provide support for abstracting existing RIA technologies and to generate rich web systems, they lack in bridging the fluid nature of the user interaction in RIAs to the design. So, only partially they can be used to transform the UX of LAs to RIAs preventing interface flaws. To meet this challenge, we proposed the use of Rich-IDM [5] and the MIGROS tools as a restructuring bridge between a flat reverse engineering of legacy user interfaces and a structured forward engineering of the presentation layer of RIAs.

\section{Tool Chain for Automatic Transformation}

The MIGROS tools exploit the OMG's ADM framework. Whereas other research groups (as those at Microsoft's and IBM's laboratories) are working on tools for the server side of existing LAs adopting horizontal transformations (paths 1 and 2 of Fig. 
1a) or vertical ones (path 3 of Fig. 1a), we focused our attention on a tool chain to refactor the UX of the existing solution. Following the path 4 of Fig. 1a: (i) we represent the UX of the source code using an extension (WAE+) of the Conallen's UML stereotypes [3]; (ii) we abstract and re-design the UX employing the Rich-IDM primitives, which give the opportunity to add knowledge on UX requirements and communication; and (iii) we map the Rich-IDM primitives to RUX elements, which have the advantage of being mechanically mappable to the target client technology (enabling a generative approach). To refactor the UX, we mainly lever on the following Rich-IDM primitives: RIA-Page Element, an atomic fragment of RIA page, which displays contents and links as directly mapped from LA's database; UX Core, a connected composition of page elements, which communicates the semantic nucleus of what is offered to the user at a given moment; Context View, a set of UX Cores, which maintains navigational context, orientation, organic, and fluid transition between the cores.

Fig. 1b draws the generation process - made up of three phases - of the MIGROS tool chain (domain and application levels) and the supporting technology (lower level). Following the diagram from left to right, in the Knowledge Extraction phase two Knowledge Extractor tools (implemented as Modisco Code2Model plug-ins) obtain an OWL domain knowledge model from the LA's database and a WAE+ application model from the LA's user interface. In the Knowledge Recomposition phase, the Knowledge Recomposer tool (written as ATL Model2Model rules) transforms the UX

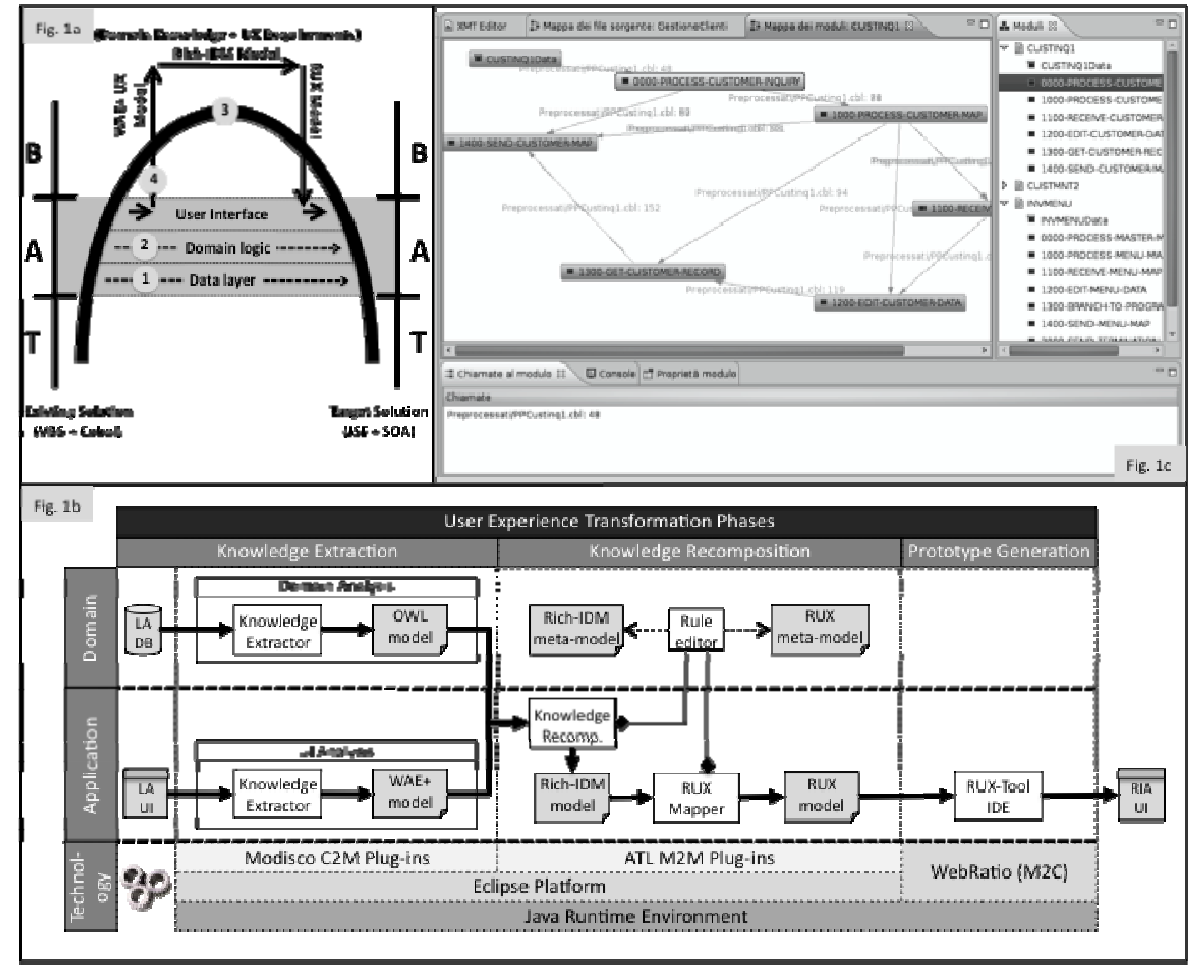

Fig. 1. MIGROS transformation process (1a), generation process (1b), and screenshot (1c) 
of the LA representing it as an OWL-encoded Rich-IDM model. To do this, the Knowledge Recomposer uses all the concepts discovered from the database and from the logical and physical user interface of the source LA. Moreover, through a Rule Editor, the Knowledge Recomposer allows an UX expert to manually improve the obtained Rich-IDM model in order to better bridge the smooth nature of the user interaction in RIAs, applying usability principles. Then, through a RUX Mapper tool, the Rich-IDM model is converted to a RUX Abstract Interface model. In this step also, the UX designer can use the Rule Editor to obtain the better mapping between RichIDM primitives and RUX elements. Finally, in the Prototype Generation phase, starting from the RUX Abstract Interface model and using the RUX-Tool IDE, and the WebRatio M2C generator, a RIA prototype of the LA's user interface can be automatically obtained. Fig. 1c shows a screenshot of the Knowledge Extractor tool. Demonstrators of MIGROS tools are available at www.migrosproject.unisalento.it.

\section{Conclusions and Future Work}

The proposed demonstration illustrates MIGROS, a tool chain based on the Eclipse platform for the model-driven modernization of the user interface of LAs. The demonstration shows how an intensive use of knowledge extraction and model transformation can help developers to automatically re-factor the UX of LAs. The originality of our approach lies in the ability to connect the transformed user interface model to UX requirements, adopting the Rich-IDM method we presented at the 13th IEEE Symposium on Web Systems Evolution. Currently we are working on a large-scale industrial innovation project, which goal is to migrate a complex LA (VB6 + Cobol) to a RIA (JSF + SOA). In the future, we will extend the tool chain to accept input from other LA's client technologies than VB6 (e.g. Cobol CICS or PowerBuilder).

Acknowledgments. This research has been partially supported by the GPS Italian funded DM29255 MIGROS project and Data Management S.p.A.

\section{References}

1. Bhallamudi, P., Tilley, S., Sinha, A.: Migrating a Web-based application to a service-based system - an experience report. In: Proceedings of the 11th IEEE International Symposium on Web Systems Evolution, WSE 2009, pp. 71-74. IEEE, NY (2009)

2. Brambilla, M., Preciado, J.C., Linaje, M., Sanchez-Figueroa, F.: Business Process -based Conceptual Design of Rich Internet Applications. In: Proceedings of the 8th International Conference on Web Engineering, ICWE 2008, pp. 155-161. IEEE, NY (2008)

3. Casteleyn, S., Daniel, F., Dolog, P., Matera, M.: Engineering Web Applications. In: Carey, M.J., Ceri, S. (eds.), pp. 978-973. Springer (2009) ISBN 978-3-540-92200-1

4. Feng, C., Hongji, Y., Hong, Z., Bing, Q., Huifang, D.: Web-based system evolution in model driven architecture. In: Proceedings of the 10th IEEE International Symposium on Web Systems Evolution, WSE 2008, pp. 69-72. IEEE, NY (2008)

5. Pandurino, A., Bolchini, D., Mainetti, L., Paiano, R.: Rich-IDM: Extending IDM to Model Rich Internet Applications. In: Proceedings of the ACM 12th Information Integration and Web-based Applications \& Services Conf., iiWAS 2010, pp. 145-152 (November 2010) 